\title{
The use of ultrasonic pulse velocity to estimate the water permeability of concretes
}

\section{Contribution à l'estimation de la perméabilité à l'eau des bétons par les vitesses des ultrasons}

\author{
Benouis Abdelhalim ${ }^{1}$, Mehamdia Ali ${ }^{1}$ \\ ${ }^{1}$ Laboratoire de Génie Civil et d'Hydraulique, Université 8 mai 1945 Guelma (24000), Algérie
}

\begin{abstract}
In this work we investigated the possibility of estimating the water permeability of concrete from the ultrasonic surface wave velocity (Rayleigh's waves). This is a method for the non-destructive permeability diagnosis of the in situ auscultation of a structure. Four ordinary concrete compositions with different W/C ratios and two self compacting concretes SCC were used. This study showed a decrease in of ultrasonic pulse velocity with the increase in the W/C ratio, this is due to the increase in porosity. Curing in air of the concrete specimens produces greater permeability than curing in water. The increase in the permeability with the increase of W/C ratio is more important for curing in water than for the curing in air. SCC1 has a lower permeability than that of SCC2, this difference is respectively $20 \%$ and $10 \%$ for curing in air and in water. The study show that permeability estimation with ultrasonic surface waves is more reliable for curing in water mode than tin curing in air. The correlations obtained between the permeability and the indirect ultrasonic velocity are linear, with an inversely proportional relation.
\end{abstract}

Résumé : Le présent travail consiste à estimer la perméabilité à l'eau par des mesures des vitesses ultrasonores des ondes de surface (ondes de Rayleigh) pour différentes compositions du béton. Ceci dans le but d'un diagnostic non destructif de l'auscultation in-situ d'ouvrage en se substituant aux carottages nécessaires aux essais traditionnels de perméabilité. Quatre compositions de béton ordinaire de différents rapports E/C et deux bétons autoplaçants avec deux ajouts différents ont été utilisés. Cette étude a montré une diminution de la vitesse de surface quand le rapport E/C augmente, ceci est dû à l'augmentation de la porosité. La vitesse des ultrasons pour le BAP1 (fillers calcaires) est 15\% supérieure à celle du BAP2 (laitier de hauts fourneaux). La perméabilité des éprouvettes conservées à l'air libre est plus importante que celles conservées dans l'eau. L'augmentation de la perméabilité avec l'accroissement du rapport E/C est plus importante pour les éprouvettes conservées dans l'eau que pour les éprouvettes conservées à l'air libre. Le BAP1 présente une perméabilité inferieure à celle du BAP2, cette différence est respectivement de $20 \%$ et $10 \%$ pour les éprouvettes conservées dans l'eau et à l'air libre. Les relations entre les vitesses ultrasoniques et les perméabilités ont montré que l'estimation de la perméabilité à partir des vitesses des ultrasons est plus fiable pour les éprouvettes conservées dans l'eau que celles à l'air libre. Ces relations sont de type linéaires avec une évolution inversement proportionnelle de la perméabilité en fonction des vitesses des ultrasons. 


\section{Introduction}

La détérioration du béton résulte en général de la pénétration d'agents agressifs à travers sa peau, entraînant des dommages irréversibles non seulement à sa surface mais également dans sa structure interne. Donc les bétons employés, aussi bien pour la construction d'ouvrages d'art que pour le stockage des déchets radioactifs, devraient présenter une perméabilité faible. Or, il est admis que la perméabilité d'un matériau est un indicateur fiable de sa durabilité [1].

La perméabilité du béton représente la capacité de ce matériau poreux à être traversé par un fluide sous un gradient de pression. Le flux se produit dans un réseau poreux, comportant des pores initiaux ainsi que dans des vides ou des fissures. Celles-ci, induites par diverses sollicitations, interconnectent généralement les chemins de percolation et diminuent ainsi la résistance du milieu au flux, ce qui provoque une augmentation de la perméabilité. La perméabilité est un paramètre pouvant influencer indirectement la durabilité des structures en béton en gouvernant la vitesse de pénétration d'agents agressifs [2].

Les méthodes ultrasonores sont utilisées depuis les années 1940 pour évaluer le béton. Le principe de ces méthodes est d'estimer d'une façon quantitative les propriétés des matériaux par des mesures de la vitesse et de l'atténuation de l'onde ultrasonore dans une gamme de fréquence donnée. Ces techniques sont largement utilisées pour quantifier les propriétés élastiques des matériaux, ainsi que pour la détection des cavités et des fissures. Plusieurs travaux de recherche ont été menés pour étudier la relation entre les propriétés du béton et les propriétés ultrasonores des ondes acoustiques. Ces études ont mis en évidence la sensibilité des paramètres de propagation à la structure poreuse du béton. Cette méthode permet de mesurer le temps de propagation d'une impulsion ultrasonique traversant le béton [3 - 4].

Le présent travail consiste à estimer la perméabilité à l'eau par des mesures des vitesses ultrasonores des ondes de surface (Onde de Rayleigh) pour différentes compositions du béton. Ceci dans le but d'un diagnostic non destructif de l'auscultation insitu d'ouvrage en se substituant aux carottages nécessaires aux essais traditionnels de perméabilité. Quatre compositions de béton ordinaire de différents rapports E/C et deux bétons auto plaçant avec deux ajouts différents ont été utilisé dans cette étude.

\section{Méthode expérimentale}

\subsection{Compositions des bétons}

Les matériaux utilisés pour la composition des différents bétons sont :

- un ciment CPJ-CEM II/A 42.5,

- un gravier concassé de nature calcaire de dimension $5 / 15 \mathrm{~mm}$,

- deux sables 0/5 mm (sable de carrière, sable roulé fin),

- un superplastifiant «MEDAPLAST SP40» fabriqué par Granitex,

- deux ajouts (fillers calcaires, laitier de haute fourneaux).

Les compositions des différents bétons et leurs résistances à 28 jours (trois éprouvettes pour chaque composition) sont mentionnées dans le tableau 1.

Tableau 1. Compositions des bétons

\begin{tabular}{|l|cc|c|cc|c|}
\hline $\begin{array}{c}\text { Matériaux } \\
\text { (kg) }\end{array}$ & BO 1 & BO 2 & BO 3 & BO 4 & BAP 1 & BAP 2 \\
\hline $\begin{array}{c}\text { Ciment } \\
\text { E au }\end{array}$ & $\mathbf{4 0 0}$ & $\mathbf{4 0 0}$ & $\mathbf{4 0 0}$ & $\mathbf{4 0 0}$ & $\mathbf{4 0 0}$ & $\mathbf{4 0 0}$ \\
\hline $\begin{array}{c}\text { Sable } \\
\text { carrière }\end{array}$ & $\mathbf{8 1 3}$ & $\mathbf{8 1 3}$ & $\mathbf{8 1 3}$ & $\mathbf{8 1 3}$ & $/$ & $/$ \\
\hline $\begin{array}{c}\text { Gravier } \\
\mathbf{5} / \mathbf{1 5}\end{array}$ & $\mathbf{9 4 6}$ & $\mathbf{9 4 6}$ & $\mathbf{9 4 6}$ & $\mathbf{9 4 6}$ & $\mathbf{8 6 5}$ & $\mathbf{8 6 5}$ \\
\hline $\begin{array}{l}\text { Sable fin } \\
\text { roulé }\end{array}$ & $/$ & $/$ & $/$ & $/$ & $\mathbf{7 6 5}$ & $\mathbf{7 6 5}$ \\
\hline $\begin{array}{l}\text { Fillers } \\
(\mathbf{2 0} \%)\end{array}$ & $/$ & $/$ & $/$ & $/$ & $\mathbf{8 0}$ & $/$ \\
\hline $\begin{array}{l}\text { Laitier } \\
(20 \%)\end{array}$ & $/$ & $/$ & $/$ & $/$ & $/$ & 80 \\
\hline E/C & 0.52 & 0.55 & 0.60 & 0.65 & 0.50 & 0.50 \\
\hline G/S & 1.16 & 1.16 & 1.16 & 1.16 & 0.88 & 0.88 \\
\hline $\begin{array}{l}\text { Résistance } \\
\text { (MPa) }\end{array}$ & $\begin{array}{c}29.39 \\
\pm 2.10\end{array}$ & $\begin{array}{c}25.86 \\
\pm 2.09\end{array}$ & $\begin{array}{c}21.80 \\
\pm 0.80\end{array}$ & $\begin{array}{c}18.98 \\
\pm 0.82\end{array}$ & $\begin{array}{c}25.20 \\
\pm 3.85\end{array}$ & $\begin{array}{c}26.45 \\
\pm 0.72\end{array}$ \\
\hline
\end{tabular}

\subsection{Mesure de la vitesse ultrasonore de l'onde de surface}

La vitesse de l'onde de surface (onde de Rayleigh) est mesurée en utilisant un générateur d'impulsions ultrasonores et deux transducteurs (émetteur, récepteur) de fréquence $54 \mathrm{kHz}$ de diamètre $49.5 \mathrm{~mm}$ qui sont mis contact avec la surface des éprouvettes par l'intermédiaire d'un couplant. L'émetteur est fixe, le récepteur se déplace à pas constant $(15 \mathrm{~cm})$ (fig.1). Les vitesses ont été déterminées par des mesures du temps d'une impulsion traversant la surface du béton [5].

Les mesures ultrasonores des ondes de surface ont été réalisées sur des poutrelles de dimensions $15 \times 15 \times 100$ $\mathrm{cm}^{3}$ (3 poutrelles pour chaque composition).

La vitesse des ondes de surface est estimée à partir de la pente de la courbe linéaire présentée sur la figure 2 
qui donne la variation du temps (acquisition du signal) en fonction de la distance (entre l'émetteur et le récepteur)

$$
V=d=\frac{\Delta y}{\Delta t}
$$

Où $d$ est la pente de la droite qui donne la variation du temps (acquisition du signal) en fonction de la distance (entre l'émetteur et la récepteur) [3].

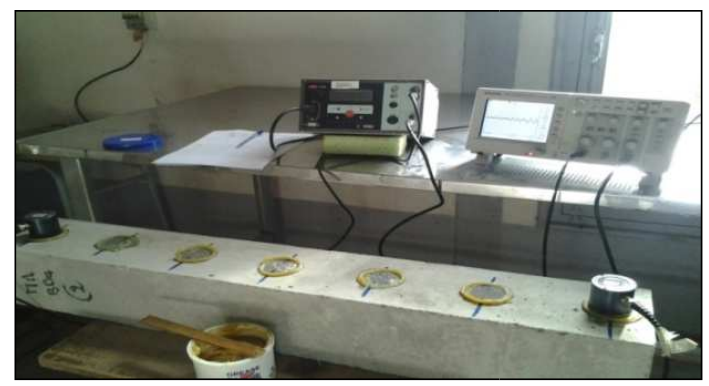

Fig. 1. Dispositif pour la mesure des paramètres de l'onde de surface

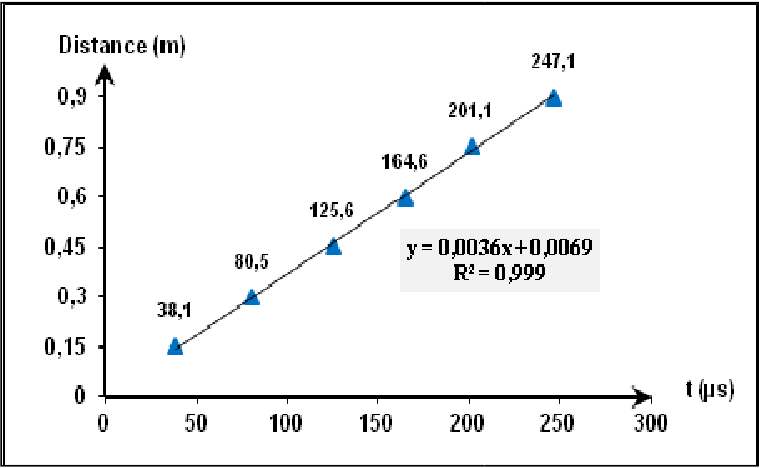

Fig. 2. Estimation de la viltesse des ondes de surface

\subsection{Mesure de la perméabilité à l'eau}

Les essais de perméabilité à l'eau ont été effectués suivant la norme européenne EN 12390-8, cette méthode consiste à déterminer la profondeur de pénétration d'eau sous pression dans un béton durci conservé dans l'eau (EN, 2012) [6].

L'essai a été réalisé sur trois éprouvettes cubiques $\left(15 \times 15 \times 15 \mathrm{~cm}^{3}\right)$ pour chaque composition de béton à 28 jours avec deux modes de conservation (à l'air libre et dans l'eau). Les perméabilités des éprouvettes conservées dans l'eau et à l'air libre sont étroitement liées, donc ceci nous permet de corréler l'une ou l'autre aux mesures indirectes par les ultrasons. Le montage nécessaire à la réalisation de cet essai est présenté par la figure 3. L'essai consiste à appliquer une pression d'eau de $5 \pm 0.5$ bar sur la surface des éprouvettes pendant une période de 72 heures, après la rupture des éprouvettes par fendage, on mesure la profondeur de pénétration d'eau $(\mathrm{H})$. L'appareillage utilisé pour mesurer la perméabilité est de type Controlab [7].

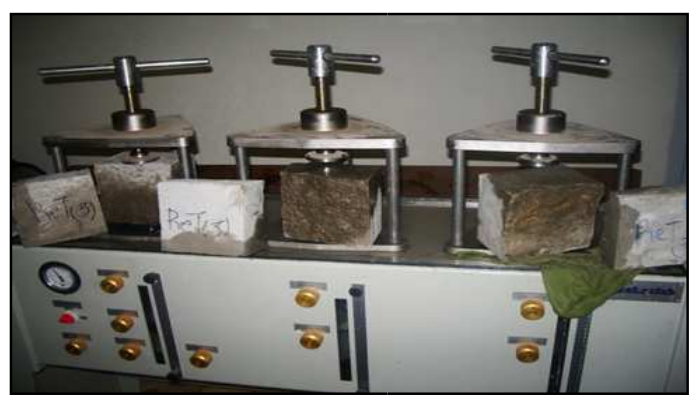

Fig. 3. Appareillage pour la mesure de la perméabilité à l'eau (profondeur de pénétration d'eau)

\section{Résultats et interprétations}

Tableau2. Vitesses des ondes de surface et perméabilités pour les différents bétons.

\begin{tabular}{|c|c|c|c|c|c|}
\hline & Béton & $\begin{array}{ll}\mathrm{BOl} & \mathrm{B} 02\end{array}$ & B03 & B04 BAPl & BAP2 \\
\hline & $\mathrm{E} / \mathrm{C}$ & $0.52 \quad 0.55$ & 0.60 & $\begin{array}{ll}0.65 & 0.50\end{array}$ & 0.50 \\
\hline & $V(\mathrm{~m} / \mathrm{s})$ & $\begin{array}{cc}3634 & 3534 \\
\pm 100 & \pm \\
300\end{array}$ & $\begin{array}{l}3200 \\
\pm 200\end{array}$ & $\begin{array}{cc}3167 & 3766 \\
\pm & \pm 300 \\
100 & \pm\end{array}$ & $\begin{array}{l}3200 \\
\pm 100\end{array}$ \\
\hline \multirow[t]{2}{*}{$\mathrm{H}(\mathrm{cm})$} & $\begin{array}{l}\text { conservation à } \\
\text { l'air }\end{array}$ & $\begin{array}{cc}14.53 & 14.8 \\
\pm 1.0 & \pm \\
& 0.6\end{array}$ & $\begin{array}{c}15 \\
\pm 0.0\end{array}$ & $\begin{array}{cc}15 & 11.3 \\
\pm 0.0 & \pm 2.0\end{array}$ & $\begin{array}{r}12.5 \\
\pm 1.2\end{array}$ \\
\hline & $\begin{array}{c}\text { conserration } \\
\text { dansl'eau }\end{array}$ & $\begin{array}{cc}0.17 & 0.7 \\
\pm 0.1 & \pm 0.4\end{array}$ & $\begin{array}{c}1,3 \\
\pm 0.8\end{array}$ & $\begin{array}{cc}1.4 & 1.2 \\
\pm 0.3 & \pm 0.2\end{array}$ & $\begin{array}{c}1.5 \\
\pm 0.3\end{array}$ \\
\hline
\end{tabular}

\subsection{Influence de la composition du béton sur la vitesse des ondes de surface}

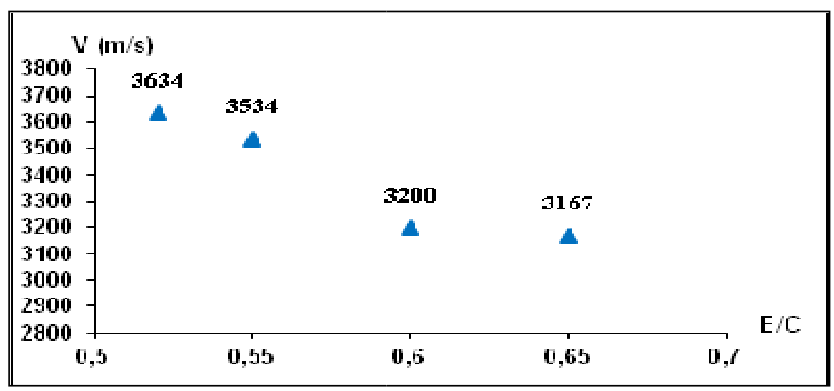

Fig. 4. . Evolution de la vitesse en fonction pour les bétons ordinaires 


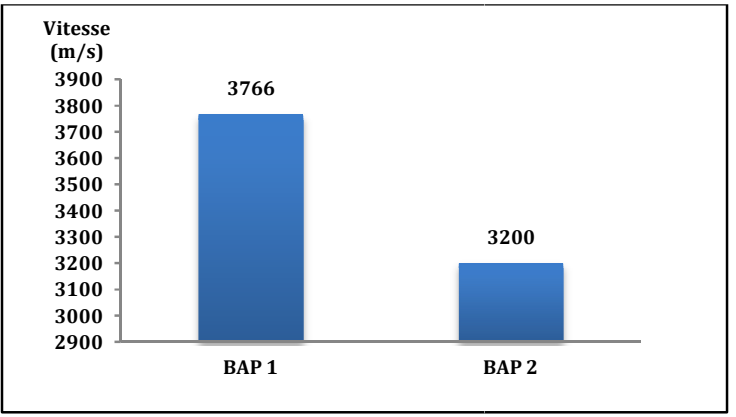

Fig. 5. . Evolution de la vitesse en fonction BAP

La figure 4 présente la variation de la vitesse de propagation de l'onde de surface en fonction du rapport E/C pour les bétons ordinaires. Les résultats montrent une diminution de la vitesse de surface quand le rapport E/C augmente et ceci dû à l'augmentation de la porosité. La diminution de la vitesse est plus importante entre le rapport $\mathrm{E} / \mathrm{C}$ variant de 0,55 à 0,60 qui se reflète par à une diminution des vitesses des ultrasons de $10 \%$ alors pour une variation de $\mathrm{E} / \mathrm{C}$ de 0,60 à 0,65 , la diminution des vitesses n'est que de $1 \%$. Donc, les vitesses des ultrasons sont beaucoup plus variables dans les bétons plastiques que dans les bétons fluides.

La figure 5 présente la variation de la vitesse de propagation de l'onde de surface pour les deux bétons autoplaçants. Les résultats montrent que la vitesse de l'onde de surface pour le BAP1 (fillers calcaires) est supérieure à celle du BAP2 (laitier de hauts fourneaux). Cette différence $(15 \%)$ n'est pas visible en termes de résistance où la différence n'excède pas $5 \%$. Ceci peut s'expliquer par la relation entre les vitesses des ultrasons et la résistance qui n'est de type linéaire.

\subsection{Influence de la composition du béton sur la perméabilité}

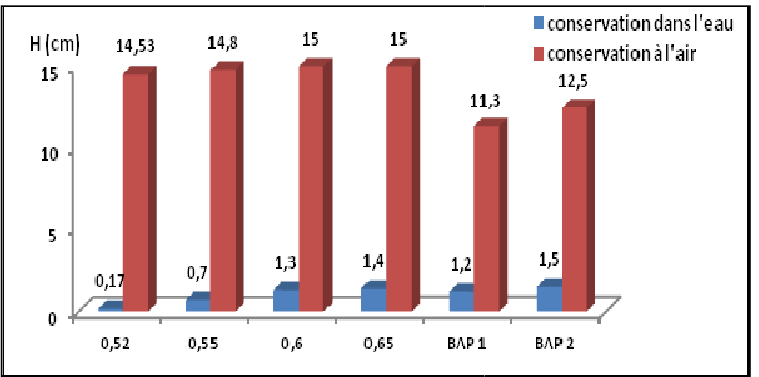

Fig. 6. Evolution de la perméabilité à l'eau (profondeur de pénétration de l'eau) pour les différents des bétons

On remarque sur cette figure que la perméabilité des éprouvettes conservées à l'air libre est plus importante que celles conservées dans l'eau. L'augmentation de la perméabilité avec l'accroissement du rapport E/C est plus importante pour les éprouvettes conservées dans l'eau que pour les éprouvettes conservées à l'air libre. Pour les éprouvettes conservées dans l'eau la profondeur de pénétration de l'eau des différents bétons reste toujours inferieure à la valeur maximale admissible pour les bétons imperméables $(5 \mathrm{~cm})$. Le BAP1 présente une perméabilité inferieure à celle du BAP2, cette différence est respectivement de $20 \%$ et $10 \%$ pour les éprouvettes conservées dans l'eau et à l'air libre.

Les résultats obtenus concordent bien avec ceux relatés dans la littérature Conz [8] a réalisé des mesures de perméabilité (profondeur de pénétration de l'eau) sur des bétons de différents rapports E/C selon la norme DIN. Il a obtenu des profondeurs d'eau de 12 , $1517 \mathrm{~mm}$ respectivement pour des rapports $\mathrm{E} / \mathrm{C}$ de $0.55,0.60,0.65 \mathrm{~mm}$. On remarque que ces résultats évoluent similairement à ceux obtenus par nos essais. il a remarqué que la profondeur de pénétration de l'eau maximal admissible $(5 \mathrm{~cm})$ est atteinte pour une rapport $\mathrm{E} / \mathrm{C}=0.70$.

\subsection{Relations entre la vitesse des ultrasons et la perméabilité}

On observe sur la figure 7 que la vitesse des ondes de surface présente une augmentation inversement proportionnelle à la perméabilité. L'estimation de la perméabilité à partir des vitesses des ultrasons est plus fiable $\left(\mathrm{R}^{2}=0.95\right)$ pour les éprouvettes conservées dans l'eau que celles à l'air libre $\left(\mathrm{R}^{2}=0.88\right)$. Ceci est du à la sensibilité de la transmission des ultrasons à l'état hygrométrique du matériau traversé, les éprouvettes conservées à l'air peuvent présenter des états hygrométriques différentes. Ces relations permettent donc une estimation de la perméabilité des bétons par ces mesures ultrasoniques 

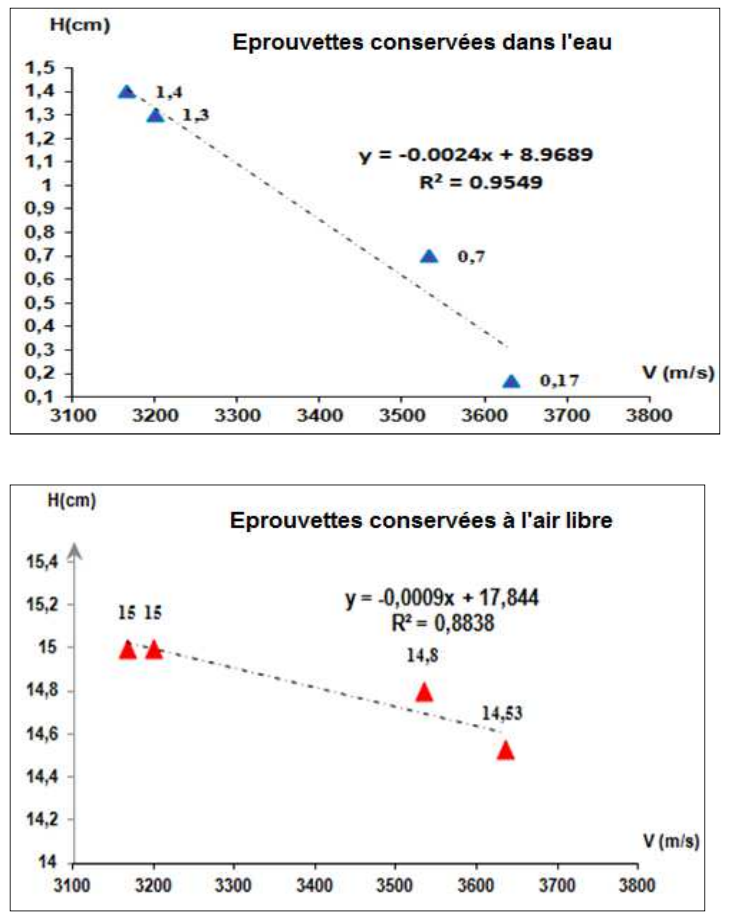

Fig. 7. Evolution de la perméabilité en fonction des vitesses des ultrasons des bétons ordinaires

\section{Conclusion}

Ce travail a été mené pour étudier la relation entre la perméabilité à l'eau et la vitesse de propagation de l'onde de surface (onde de Rayleigh) dans un matériau à matrice cimentaire. Ceci dans le but de substituer ces essais non destructifs aux essais destructifs de perméabilité réalisés sur des carottes prélevées sur les ouvrages à ausculter. Les mesures des vitesses des ultrasons ont été menées en mode de transmission surfacique pour pouvoir ausculter tous les éléments d'ouvrage (poteaux, poutres, planchers, ...) avec la facilité d'accessibilité. L'étude a concerné quatre bétons ordinaires et deux bétons autoplaçants et a mené aux conclusions suivantes :

- une diminution de la vitesse de surface quand le rapport $\mathrm{E} / \mathrm{C}$ augmente et ceci dû à l'augmentation de la porosité. La diminution de la vitesse est plus importante entre le rapport $\mathrm{E} / \mathrm{C}$ variant de 0,55 à 0,60 conduit à une diminution des vitesses des ultrasons de $10 \%$ alors pour une variation de $\mathrm{E} / \mathrm{C}$ de 0,60 à 0,65 .

- La vitesse des ultrasons pour le BAP1 (fillers calcaires) est $15 \%$ supérieure à celle du BAP2 (laitier de hauts fourneaux).

-La perméabilité des éprouvettes conservées à l'air libre est plus importante que celles conservées dans l'eau. L'augmentation de la perméabilité avec l'accroissement du rapport E/C est plus importante pour les éprouvettes conservées dans l'eau que pour les éprouvettes conservées à l'air libre. Pour les éprouvettes conservées dans l'eau la profondeur de pénétration de l'eau pour un rapport $\mathrm{E} / \mathrm{C}$ de 0,65 reste toujours inferieure à la valeur maximale admissible (5 $\mathrm{cm})$. Le BAP1 présente une perméabilité inferieure à celle du BAP2, cette différence est respectivement de $20 \%$ et $10 \%$ pour les éprouvettes conservées dans l'eau et à l'air libre.

- L'estimation de la perméabilité à partir des vitesses des ultrasons est plus fiable pour les éprouvettes conservées dans l'eau (essais normalisés) que celles à l'air libre. Ces relations sont de type linéaire avec une évolution inversement proportionnelle de la perméabilité en fonction des vitesses des ultrasons.

\section{Références}

1. Z. Lafhaj, Caractérisation des propriétés mécanique et acoustique d'un mortier dégradé chimiquement: étude expérimentale, $16^{\text {ème }}$ Congrès Français de Mécanique, Ecole centrale de Lille, France (2005).

2. M. Choinska, Etude expérimentale de l'interaction endommagement-température perméabilité du béton, Thèse de doctorat, Ecole Centrale de Nantes, France, (2006).

3. . Soltani, Etude expérimentale de la propagation de l'onde de surface dans un milieu poreux sec et saturé, COFREND2008, Toulouse, France, (2008).

4. M. Chekroun, Diffusion multiple des ultrasons pour l'evaluation non destructive du beton avec des ondes de surface, COFREND2008, Toulouse, France, (2008).

5. Controls, Instruction manual : Ulrasonic pulse velocity tester, Mod. 58-E0048, Italie, (2002).

6. EN 12390-8, Essai pour béton durci : Partie 8 Profondeur de pénétration d'eau sous pression, AFNOR, France, (2012).

7. Controlab, Manuel d'utilisation Appareil pour mesurer la perméabilité selon la norme EN 123908 (AFNOR), Réf.E0651. France (2008).

8. D. Conz, Transport d'humidité dans les éléments de construction en béton imperméable à l'eau (béton WU)", rapport d'étude, Zeugin Bauberatungen AG, Münsingen, Allemagne, (2006).. 